\title{
Hidatidosis esplénica. Resultados de una serie de casos consecutiva intervenidos quirúrgicamente
}

\author{
Splenic hydatidosis. Results of a series of consecutive cases undergoing surgery
}

\author{
Carlos Manterola1 y Nataniel Claros ${ }^{2}$
}

'Departamento de Cirugía y Centro de Estudios Morfológicos y Quirúrgicos (CEMyQ), Universidad de La Frontera, Chile.
'Servicio de Cirugía, Hospital Obrero N¹, Caja Nacional de Salud, La Paz, Bolivia.

Parcialmente financiado por el proyecto DI19-0030, Universidad de La Frontera.

Conflictos de interés: Ninguno.

Recibido: 27 de julio de 2020 (segunda versión: 6 de marzo de 2021) / Aceptado: 7 de marzo de 2021

\section{Resumen}

Introducción: La echinococcosis esplénica (EE), suele ser una enfermedad asintomática cuyo diagnóstico se plantea de forma incidental. Objetivo: Determinar morbilidad post-operatoria (MPO) y recurrencia en pacientes intervenidos quirúrgicamente por EE. Material y Método: Serie de casos con seguimiento, de pacientes con EE intervenidos de forma consecutiva, entre 2000 y 2018. Las variables resultado fueron MPO y recurrencia. Otras variables de interés fueron: diámetro y localización del quiste, tipo de cirugía, tiempo quirúrgico, estancia hospitalaria, y mortalidad. Los pacientes fueron seguidos de forma clínica y con imágenes por un mínimo de 18 meses. Se utilizó estadística descriptiva, con medidas de tendencia central y dispersión. Resultados: Se intervinieron 26 pacientes $(53,8 \%$ hombres), con una mediana de edad de 41,5 años. Las medianas del diámetro de los quistes, el tiempo quirúrgico y el tiempo de hospitalización fueron $14,5 \mathrm{~cm}$; 65 minutos y 4,5 días, respectivamente. La MPO fue $11,5 \%$ ( 3 casos). No hubo mortalidad. Con una mediana de seguimiento de 94 meses, se verificó una recurrencia de 3,8\%. Conclusión: Los resultados verificados, en términos de MPO, mortalidad y recurrencia son apropiados en comparación a otras series publicadas.

Palabras clave: echinococcosis, hidatidosis esplénica; quiste hidatídico del bazo; esplenectomía.

\begin{abstract}
Background: Splenic echinococcosis (SE) is usually an asymptomatic disease whose diagnosis is made incidentally. Aim: To determine postoperative morbidity (POM) and recurrence in patients who underwent surgery for SE. Methods: Case series with followup, of patients with SE operated on, consecutively, between 2000 and 2018. The outcome variables were POM and recurrence. Other variables of interest were diameter and location of the cyst, type of surgery, surgical time, hospital stay, and mortality. The patients were followed up clinically and with images for a minimum of 18 months. Descriptive statistics were used, with measures of central tendency and dispersion. Results: Twenty-six patients (53.8\% men), with a median age of 41.5 years, underwent surgery in this period. The medians of cysts diameter, surgical time and hospital stay were $14.5 \mathrm{~cm}, 65 \mathrm{~min}$, and 4.5 days respectively. POM was $11.5 \%$ ( 3 cases). There was no mortality. With a median follow-up of 94 months, a recurrence of $3.8 \%$ was verified. Conclusion: Verified results, in terms of POM, mortality and recurrence are appropriate in comparison with those of series of similar size and follow-up.

Keywords: echinococcosis; cystic echinococcosis; splenectomy; splenic hydatid cyst.
\end{abstract}

\section{Correspondencia a:}

Carlos Manterola Delgado

carlos.manterola@ufrontera.cl 


\section{Introducción}

L a echinococcosis esplénica (EE), causada por la etapa larvaria de Echinococcus granulosus, ocupa el tercer lugar en frecuencia de localización ( 2 a $6 \%$ ); después del hígado (70 a $80 \%$ ) y los pulmones (15 a $20 \%)^{1-9}$.

Si bien la afectación esplénica puede ser solitaria, también puede acompañar la afectación sincrónica de otros órganos, comúnmente en hígado y peritoneo, lo que puede observarse hasta en $30 \%$ de los $\operatorname{casos}^{1,3,10-13}$.

El primer caso fue descrito en 1790 por Bertelot, y la primera serie de casos que se publicó corresponde a Sabadini ${ }^{12}$.

Suele ser una enfermedad asintomática cuyo diagnóstico se plantea de forma incidental en el curso de un estudio de imágenes. Los escasos síntomas son dolor en el cuadrante superior izquierdo del abdomen secundaria a esplenomegalia; y eventualmente distensión abdominal $^{3,9,14,15}$.

El tratamiento de la EE sigue siendo motivo de controversia, pues existe evidencia del uso de tratamiento médico, PAIR (Puncture, Aspiration, Injection, Re-Aspiration), combinaciones de ambos y cirugía. $Y$ esta última opción, con y sin preservación del bazo; esplenectomía parcial y total, esplenectomía abierta y vía laparoscópica; con y sin uso de vacuna anti-neumocócica en casos de esplenectomía total ${ }^{8,11,16,17}$.

Objetivo de este estudio fue determinar morbilidad post-operatoria (MPO) y recurrencia en pacientes intervenidos quirúrgicamente por $\mathrm{EE}$.

\section{Metodología}

El reporte de este estudio fue escrito siguiendo la propuesta MInCir para el reporte de estudios observacionales descriptivos $^{18}$.

\section{Diseño}

Serie de casos con seguimiento.

\section{Centro}

El estudio fue realizado en Clínica RedSalud Mayor Temuco, en el período enero de 2000 y diciembre de 2018 (19 años).

\section{Participantes}

Se incluyeron todos los pacientes portadores de EE, intervenidos quirúrgicamente, de forma consecutiva, en el período e institución antes señalado, por el primer autor (CM). No se consideraron exclusiones.

\section{Tamaño de muestra}

Se trabajó con la totalidad del universo de pacientes con EE intervenidos quirúrgicamente en el período e institución señalada. Por ende, no se realizó estimación de tamaño de la muestra.

\section{Protocolo de estudio}

Todos los pacientes fueron estudiados con pruebas diagnósticas generales, determinación de ELISA-IgE e IgG para E. granulosus, radiología simple de tórax, ultrasonografía abdominal y tomografía axial computarizada. Los quistes fueron clasificados de acuerdo con la propuesta de la Organización Mundial de la Salud-OMS ${ }^{19}$.

\section{Variables}

La variable resultado fue MPO (dicotomizada en sí/ no y según propuesta de Clavien \& Dindo $^{20}$, medida en meses posterior a la cirugía. Otras variables de interés fueron: mortalidad y recurrencia. Se consideraron además algunas variables clínicas como: edad, sexo, diámetro y localización del quiste, cirugía realizada, y tiempo de seguimiento.

\section{Protocolo quirúrgico}

Todos los pacientes fueron intervenidos bajo anestesia general y vía laparotómica. En todos ellos se administró profilaxis antimicrobiana con cefazolina en dosis de $1 \mathrm{~g}$ cada $8 \mathrm{~h}$, la que se mantuvo por $24 \mathrm{~h}$. La vía de acceso más frecuente fue la laparotomía media supraumbilical; en algunos casos, se recurrió a una ampliación en "J" a la izquierda ${ }^{21}$. Una vez identificado el quiste, se procedió a rodear la lesión con compresas y luego se realizó punción evacuadora del quiste y exéresis de la germinal y vesícula hijas, lavando posteriormente la cavidad con solución de polivinilpirrolidona. Posteriormente, se realizó división del ligamento espleno-cólico y maniobra de Gómez y Gómez, para liberar el bazo del diafragma. En este momento se tomó la decisión de realizar esplenectomía o periquistectomía. Cuando la indicación fue esplenectomía, se procedió a la identificación, ligadura y sección del pedículo vascular esplénico. La cirugía finalizaba con la instalación de un drenaje siliconado en el hipocondrio izquierdo que se exteriorizaba por contrabertura.

\section{Seguimiento}

El seguimiento mínimo considerado fue de 18 meses. Este, se realizó mediante el estudio de las historias clínicas y certificados de defunción obtenidos del Registro Civil e Identificación.

\section{Análisis estadístico}

Utilizando el paquete estadístico Stata 11.0, se realizó un análisis exploratorio de los datos. Se aplicó estadística descriptiva con cálculo de porcentajes, medidas de tendencia central y dispersión. 


\section{Sesgos}

Se enmascaró la recopilación de datos y se minimizaron los sesgos mediante el seguimiento completo de todos los casos durante un período mínimo de 18 meses (último caso reclutado), con excepción del fallecimiento de algún paciente.

\section{Aspectos éticos}

Se observaron las pautas éticas para la investigación en seres humanos definidas por la Declaración de Helsinki² ${ }^{22}$.

\section{Resultados}

En el período estudiado se intervinieron quirúrgicamente 26 pacientes, con una mediana de edad de 41,5 años; $53,8 \%$ eran de sexo masculino. Además, el $46,2 \%$ de la serie presentaba algún tipo de co-morbilidad (Tabla 1).

El tiempo de síntomas tuvo una mediana de siete meses (Tabla 2). La mayoría de los pacientes $(69,2 \%)$ reportaron malestar o dolor leve en el hipocondrio izquierdo; el resto eran asintomáticos. El examen físico permitió verificar la presencia de una masa palpable en el cuadrante superior izquierdo del abdomen o esplenomegalia en 16 pacientes $(61,5 \%)$.

Las pruebas de laboratorio general se encontraron dentro de valores normales y sólo destacaron los valores máximos del recuento total de leucocitos, bilirrubina total, fosfatasa alcalina y de ambas transaminasas (los que se registraron en algunos pacientes con hidatidosis hepática concomitante), así como de glicemia y creatinina en pacientes con co-morbilidades (Tabla 3 ).

El $65,4 \%$ de las lesiones eran hipoecoicas o tipo CE1 de clasificación OMS (Tabla 1). La localización más frecuente de las lesiones fue el polo inferior del bazo (10 casos; $38,5 \%$ ), seguida de compromiso total del bazo, en los que se encontró una víscera casi inexistente, comprimida por el quiste ( 7 casos; $26,9 \%$ ) (Tabla 1 ).

Por otra parte, en 11 casos $(42,3 \%)$, se verificó la existencia de quistes hidatídicos en otras localizaciones; destacando hígado y peritoneo (Tabla 1 ).

Se realizó esplenectomía en $50,0 \%$ de los casos (13 casos). En 11 casos $(56,0 \%)$, fue necesario realizar otras cirugías de forma concomitante, sobre quistes de localización hepática y peritoneal (Tabla 1).

Las medianas del diámetro de los quistes, el tiempo quirúrgico y el tiempo de hospitalización fueron $14,5 \mathrm{~cm}$; 65 min y 4,5 días, respectivamente (Tabla 2).

La MPO fue 11,5\% (3 casos), las que fueron tipo I, II y IIIb de Clavien \& Dindo (Tabla 4). No hubo mortalidad operatoria. Un paciente falleció durante el seguimiento (a los 64 meses), a los 80 años de edad, a consecuencia de una descompensación cardiovascular.
Tabla 1. Hidatidosis esplénica. Distribución de variables clínicas de los pacientes en estudio $(n=26)$

\begin{tabular}{lcr}
\hline Variable & n casos & $\%$ \\
Co-morbilidades & & \\
Ninguna & 14 & 53,8 \\
HTA & 6 & 23,0 \\
DM II & 2 & 7,6 \\
Cardiopatía & 1 & 3,9 \\
EPOC & 1 & 3,9 \\
Colelitiasis & 1 & 3,9 \\
Embarazo & 1 & 3,9
\end{tabular}

Características ultrasonográficas

$\begin{array}{lrr}\text { CE1 } & 17 & 65,4 \\ \text { CE2 } & 4 & 15,4 \\ \text { CE3 } & 3 & 11,6 \\ \text { CE4 } & 2 & 7,6\end{array}$

Otras localizaciones

$\begin{array}{lrl}\text { Sólo bazo } & 15 & 57,7\end{array}$

Hígado $\quad 5 \quad 19,2$

Peritoneo $\quad 5 \quad 19,2$

$\begin{array}{lll}\text { Renal } & 1 & 3,9\end{array}$

Localización del quiste

$\begin{array}{lrr}\text { Polo superior } & 6 & 23,0\end{array}$

$\begin{array}{lll}\text { Polo inferior } & 10 & 38,5\end{array}$

Hilio $\quad 3 \quad 11,6$

$\begin{array}{lll}\text { Totalidad del bazo } & 7 & 26,9\end{array}$

Tipo de cirugía

$\begin{array}{lll}\text { Periquistectomía } & 13 & 50,0\end{array}$

$\begin{array}{lll}\text { Esplenectomía } & 13 & 50,0\end{array}$

Otras cirugías simultáneas

Ninguna

$15 \quad 57,7$

Exéresis de quistes peritoneales

Periquistectomía hepática

Periquistectomía renal

$5 \quad 19,2$

$5 \quad 19,2$

13,9

HTA: Hipertensión arterial. DM II: Diabetes mellitus tipo II. EPOC: enfermedad pulmonar obstructiva crónica.

Tabla 2. Hidatidosis esplénica. Distribución de variables clínicas de los pacientes en estudio $(n=26)$

\begin{tabular}{lcc}
\hline Variable & Mediana & Mínimo - Máximo \\
Edad (años) & 41,5 & $18-75$ \\
Diámetro del quiste (cm) & 14,5 & $5-30$ \\
Tiempo síntomas (meses) & 7 & $4-10$ \\
Tiempo quirúrgico (min) & 65 & $50-90$ \\
Hospitalización (días) & 4,5 & $2-22$ \\
Seguimiento (meses) & 94 & $18-206$
\end{tabular}


De los pacientes esplenectomizados, en siete (53,8\%), se administró vacuna anti-neumocócica; en tres de ellos (23,1\%), se verificó bazo supernumerario que se conservó; y en los tres restantes no se realizó tratamiento adicional alguno.

Al desagrupar los casos tratados con esplenectomía $(n=13)$ vs. periquistectomía esplénica $(n=13)$; no se verificaron diferencias significativas en términos de MPO (un caso para el sub-grupo de esplenectomía vs. dos casos para el grupo periquistectomía).

Con una mediana de seguimiento de 94 meses, se verificó una recurrencia de 3,8\%. Esto ocurrió en un pa-

Tabla 3. Hidatidosis esplénica. Distribución de variables de laboratorio en los pacientes en estudio $(n=26)$

\begin{tabular}{lcc}
\hline Variable & Mediana & Mínimo - Máximo \\
\hline Hematocrito $(\%)$ & 39,5 & $31-47$ \\
Recuento de leucocitos $(x \cup L)$ & 8450 & $6.000-15.600$ \\
\hline Recuento de plaquetas $(x \cup L)$ & 200 & $140-350$ \\
Creatinina ( $\mathrm{mg} / \mathrm{dL})$ & 93 & $0,6-1,5$ \\
Glicemia $(\mathrm{mg} / \mathrm{dL})$ & 1,0 & $80-150$ \\
Bilirrubina total $(\mathrm{mg} / \mathrm{dL})$ & 0,9 & $0,5-1,3$ \\
Fosfatasas alcalinas $(\mathrm{U} / \mathrm{L})$ & 207 & $100-840$ \\
ASAT $(\mathrm{U} / \mathrm{L})$ & 19 & $8-143$ \\
ALAT $(\mathrm{U} / \mathrm{L})$ & 20 & $5-182$ \\
Protrombina $(\%)$ & 90 & $55-100$ \\
\hline
\end{tabular}

ciente con hidatidosis esplénica y peritoneal concomitante (tratado con periquistectomía esplénica), y la recidiva fue a nivel peritoneal (Tabla 4). Fue diagnosticada a los 48 meses del postoperatorio y reintervenida a los dos meses de su diagnóstico.

Tabla 4. Hidatidosis esplénica. Morbilidad post-operatoria de los pacientes en estudio $(n=26)$

\begin{tabular}{lcc}
\hline Variable & $\mathbf{n}$ casos & $\%$ \\
MPO & & \\
Sí & 3 & 11,5 \\
No & 23 & 88,5
\end{tabular}

Causas de MPO

Atelectasia $\quad 1 \quad 3,9$

ISO $\quad 1 \quad 3,9$

Eventración $\quad 1 \quad 3,9$

MPO según Clavien

$\begin{array}{lll}\text { I } & 1 & 3,9 \\ \text { II } & 1 & 3,9 \\ \text { IIb } & 1 & 3,9\end{array}$

Recurrencia

$\mathrm{Si}^{*} \quad 1 \quad 3,9$

No $25 \quad 96,1$

MPO: Morbilidad post-operatoria. ISO: Infección del sitio operatorio. *Recurrencia peritoneal, en paciente con hidatidosis esplénica y peritoneal concomitante.

Tabla 5. Hidatidosis esplénica. Resumen de las series más numerosas publicadas desde 1990

\begin{tabular}{|c|c|c|c|c|c|c|c|c|}
\hline Autor (año) & País & n casos & $\begin{array}{c}\text { Esplenectomía } \\
(\%)\end{array}$ & $\begin{array}{l}\text { Hospitalización } \\
\text { (días)* }\end{array}$ & $\begin{array}{c}\text { MPO } \\
(\%)\end{array}$ & $\begin{array}{l}\text { Mortalidad } \\
\text { (\%) }\end{array}$ & $\begin{array}{c}\text { Seguimiento } \\
\text { (meses) }^{*}\end{array}$ & $\begin{array}{c}\text { Recurrencia } \\
(\%)\end{array}$ \\
\hline Tarcoveanu $(2002)^{32}$ & Rumania & 28 & 92,9 & 13,3 & 23,2 & 0,0 & $N R$ & 7,1 \\
\hline $\operatorname{Dar}(2002)^{3}$ & India & 26 & 100,0 & 8,0 & 23,1 & 3,8 & 84,0 & 3,8 \\
\hline Ousadden $(2010)^{31}$ & Marruecos & 23 & 39,1 & 23 & 34,8 & 0,0 & NR & 8,7 \\
\hline Ben Ameur $(2015)^{16}$ & Túnez & 21 & 57,1 & NR & 9,5 & 0,0 & 36,0 & 0,0 \\
\hline Culafic $(2010)^{28}$ & Serbia & 20 & 65,0 & NR & 10,0 & 5,0 & $N R$ & $N R$ \\
\hline Atmatzidis $(2003)^{39}$ & Grecia & 19 & 57,9 & 16,0 & 26,3 & 5,2 & 52,0 & 15,8 \\
\hline Ozogul (2015) & Turquía & 17 & 70,6 & 9,2 & 17,6 & 5,9 & $N R$ & $N R$ \\
\hline Eris $(2013)^{15}$ & Turquía & 16 & 100,0 & 4,0 & 12,5 & 0,0 & NR & 0,0 \\
\hline Herrera Merino $(1991)^{5}$ & España & 16 & 100,0 & NR & 25,0 & 0,0 & NR & $N R$ \\
\hline Durgun $(2003)^{14}$ & Turquía & 14 & 78,6 & 9,8 & 35,7 & 0,0 & NR & 0,0 \\
\hline Ozdogan $(2001)^{8}$ & Turquía & 14 & 85,7 & NR & 28,6 & 0,0 & NR & NR \\
\hline Safioleas $(1997)^{29}$ & Grecia & 14 & 78,6 & 15,0 & 21,4 & 7,1 & $N R$ & 0,0 \\
\hline Arikanoglu (2012) & Turquía & 11 & 72,7 & 8,7 & 36,4 & 0,0 & 26,4 & NR \\
\hline
\end{tabular}

*Promedio de las series. NR: No reportado. Existen series numéricamente interesantes de EE, cuyos resultados están mezclados con hidatidosis de otras localizaciones; y los autores no desagruparon los datos por localización, por lo que no pudieron ser incorporados en esta tabla ${ }^{4,6}$. 


\section{Discusión}

\section{Comentarios sobre los resultados obtenidos}

La equinococcosis representa aproximadamente el $65 \%$ de las lesiones quísticas del bazo ${ }^{2,7,13}$. Sin embargo, la localización esplénica representa sólo entre 0,5 y $8 \%$ de todas las localizaciones de la equinococcosis humana ${ }^{2,7,23}$.

El principal problema en el diagnóstico de la EE es diferenciarla de otras lesiones quísticas del bazo, cuyo aspecto imagenológico puede ser similar, como quiste epidermoide, absceso, hematoma, neoplasia quística del bazo, etc. ${ }^{9,24}$.

Dada la escasa sintomatología, la EE suele cursar asintomática por largos períodos (hasta 20 años), siendo su diagnóstico habitualmente producto de un hallazgo en un estudio de imágenes, lo que explica su gran diámetro en una importante proporción de los casos ${ }^{12,25-27}$. Esto fue similar a lo verificado en nuestros pacientes, en los que tuvimos $30,8 \%$ de diagnóstico por hallazgos y con una mediana de diámetro de $14,5 \mathrm{~cm}$ (con $50 \%$ de los quistes con diámetros sobre los $14,5 \mathrm{~cm}$ ).

Los hallazgos ultrasonográficos suelen ser de masas únicas, homogéneamente anecoicas. Ocasionalmente, tienen patrón sólido, el que puede corresponder a membranas infectadas. En la tomografía axial computarizada se suelen apreciar lesiones de menor atenuación que el bazo circundante, ocasionalmente con focos de calcificación. Además, proporciona información adicional (como otras localizaciones de hidatidosis) ${ }^{24}$. En nuestra experiencia constatamos que $65,4 \%$ de los quistes eran lesiones anecoicas.

La concomitancia con otras lesiones hidatídicas abdominales se describe hasta en $30 \%^{1,3,10-13,27}$; en nuestra experiencia fue de $42,3 \%$.

El tratamiento sigue siendo quirúrgico, destacando el rol de la esplenectomía (ya sea total o parcial), la evidencia la avala en términos de número de pacientes intervenidos $\mathrm{y}$ por los resultados obtenidos en términos de MPO, mortalidad y recurrencia ${ }^{1,3,5,8,11,14,15,25,28,29}$. Un resumen de las series más numerosas se puede apreciar en la Tabla 5. En ésta se puede constatar que la mayor parte de las series realizaron esplenectomía en mas de $70 \%$ de los casos, lo que contrasta con nuestra experiencia, pues recurrimos a la exéresis del bazo sólo en $56 \%$ de los casos. Existen series numéricamente interesantes de EE, cuyos resultados fueron mezclados con hidatidosis de otras localizaciones. Lamentablemente, los autores no desagruparon los datos por localización, por lo que no pudieron ser incorporados en esta tabla ${ }^{4,6}$.

Respecto de MPO, mortalidad y recurrencia, en nuestros pacientes constatamos 12,$0 ; 0,0$ y 4,0\%, respectivamente (con una mediana de seguimiento de 95 meses), cifras que se pueden considerar muy adecuadas respecto de las series de mayor tamaño (Tabla 5), en las

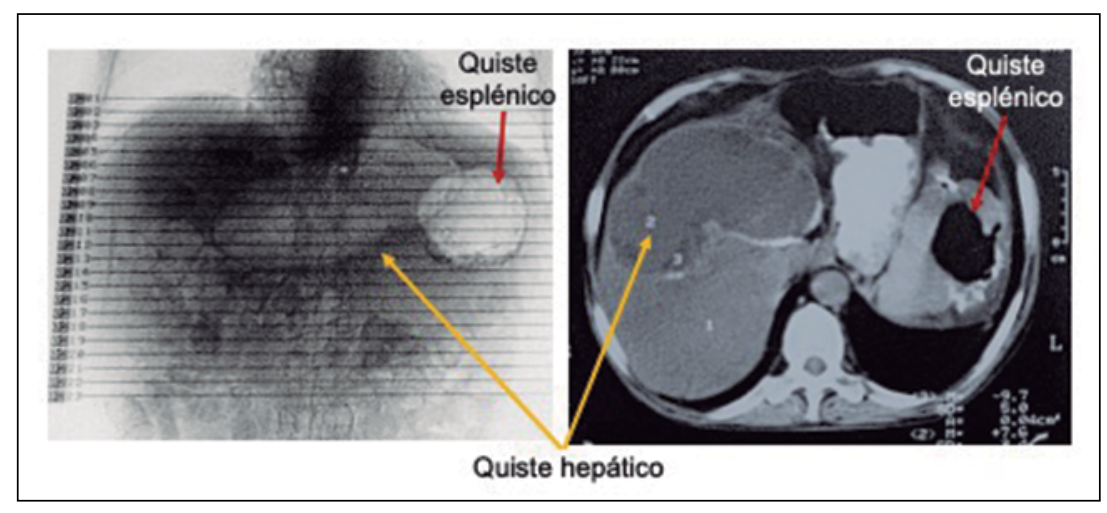

Figura 1. Imágenes tomográficas de paciente con quiste hidatídico hepático y esplénico (este último abscedado).
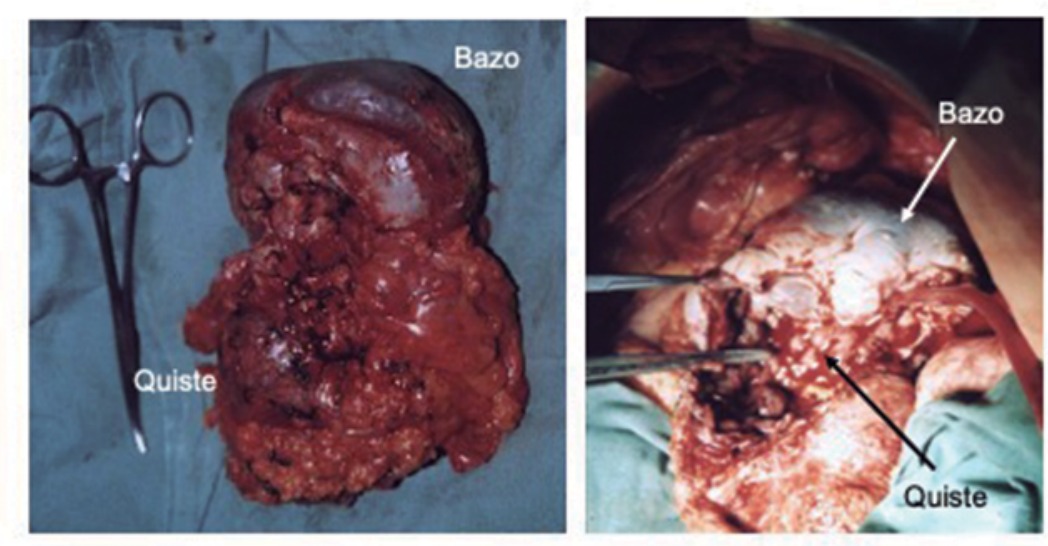

Figura 2. Especímen quirúrgico de quiste esplénico y bazo (A). Imagen quirúrgica de paciente con gran quiste esplénico abscedado drenado (B).

que nueve de las 13, reportaron MPO entre 23,1 y $36,4 \%$; cinco de las 13 , reportaron mortalidades entre 3,8 y $7,1 \%$ y cinco de las 13 no reportan su recurrencia. Y de las ocho restantes (la mayoría con seguimientos muy cortos), en cuatro series se reportan recurrencias entre 3,8 y $15,8 \%$.

Existe evidencia que la esplenectomía no se asocia a mayor MPO, mortalidad ni recurrencia respecto de tratamiento conservador ${ }^{15,30}$; por el contrario, hay algunas series comparativas en las que se constató lo opuesto $0^{8,31,32}$.

Por otra parte, cabe señalar, que existe experiencia con esplenectomía parcial laparoscópica ${ }^{33}$ y robótica ${ }^{34}$; pero se trata sólo de reportes de casos aislados, con escaso seguimiento y conversiones de hasta $50 \%{ }^{35,36}$.

También existe evidencia de tratamientos percutáneos como cirugía con preservación esplénica en reportes de casos, con cortos períodos de seguimiento, y necesidad de esplenectomía en el post-operatorio mediato ${ }^{37,38}$. 
En general, no utilizamos quimioprofilaxis con albendazol para disminuir la probabilidad de recidiva, pre ni post-operatoria. Hace algunos años aportamos evidencia experimental respecto de la falta de eficacia al administrar quimioprofilaxis anti-parasitaria en el pre-operatorio, midiendo las concentraciones intraquísticas del sulfóxido de albendazol y la viabilidad de los protoescólex en pacientes tratados con albendazol ${ }^{39}$; y una alta recidiva observada en pacientes con echinococcosis abdominal intervenidos y pese a recibir en el post-operatorio albendazol durante tres meses ${ }^{40}$.

\section{Novedad de la propuesta}

Se trata de una casuística unicéntrica y regional, de un número considerable de casos respecto de la evidencia existente en la literatura médica, cuyos resultados en términos de MPO, mortalidad y recurrencia (con seguimientos apropiados), son adecuados considerando la magnitud y complejidad de la enfermedad.

\section{Limitaciones del estudio}

Se trata de una serie pequeña, en la que todos los pacientes fueron operados por el mismo cirujano.

\section{Conclusión}

Los resultados verificados en esta serie, en términos de MPO, mortalidad y recurrencia, son satisfactorios en comparación con los de series de tamaño y seguimiento similares.

\section{Referencias bibliográficas}

1.- Arikanoglu Z, Taskesen F, Gumus H, Onder A, Aliosmanoglu I, Gul M, et al. Selecting a surgical modality to treat a splenic hydatid cyst: total splenectomy or spleen-saving surgery? J Gastrointest Surg. 2012; 16: 1189-93. doi: 10.1007/s11605-012-1837-2.

2.- $\quad$ Amr S S, Amr Z S, Jitawi S, Annab H. Hydatidosis in Jordan: an epidemiological study of 306 cases. Ann Trop Med Parasitol. 1994; 88: 623-7. doi: 10.1080/00034983.1994.11812913.

3.- Dar M A, Shah O J, Wani N A, Khan F A, Shah P. Surgical management of splenic hydatidosis. Surg Today 2002; 32: 224-9. doi: 10.1007/ s005950200025.

4.- Hamamci E O, Besim H, Korkmaz A. Unusual locations of hydatid disease and surgical approach. ANZ J Surg. 2004; 74: 356-60 doi: $10.1007 / \mathrm{s} 005950200025$.

5.- Herrera Merino N, Abascal Morte J, Díaz del Río Botas M, Peñalver R, Colás Vicente A, Sánchez Movilla A, Inchausti Teja J. Splenic hydatid cyst. A report of 16 cases. Rev Esp Enferm Dig. 1991; 79: 254-8. PMID: 2054212.

6.- Lianos G D, Lazaros A, Vlachos K, Georgiou G K, Harissis H V, Mangano A, et al. Unusual locations of hydatid disease: a 33 year experience analysis on 233 patients. Updates Surg. 2015; 67: 279-82. doi: 10.1007/s13304015-0291-6.

7.- Malik A A, ul Bari S, Younis M, Wani K A, Rather A A. Primary splenic hydatidosis. Indian J Gastroenterol. 2011; 30: 175-7. doi: 10.1007/ s11605-012-1837-2.

8.- Özdoğan M, Baykal A, Keşkek M, Yorgancı K, Hamaloğlu E, Sayek I. Hydatid cyst of the spleen: treatment options. Int Surg. 2001; 86: 122-6. PMID: 11918237.
9.- Rasheed K, Zargar S A, Telwani A A. Hydatid cyst of spleen: A diagnostic challenge. North Am J Med Sci. 2013; 5: 10-20 doi: 10.4103/1947-2714.106184.

10.- Manterola C, Vial M, Losada H, Fonseca F, Bustos L, Muñoz S, Barroso M. Uncommon locations of abdominal hydatid disease. Trop Doct. 2003; 33: 179-80. doi: 10.1177/004947550303300324.

11.- Ozogul B, Kisaoglu A, Atamanalp S S, Ozturk G, Aydinli B, Yıldırgan M I, Kantarcı A M. Splenic hydatid cyst: 17 cases. Indian J Surg. 2015; 77 (Suppl 2): 257-60. doi: 10.1007/ s11605-012-1837-2.

12.- Prieto M, Marquina T, Mifsut $P$, Moreno T. Splenic hydatidosis: 5 cases of this location. Enferm Infecc Microbiol Clin. 2011; 29: 634-5. doi: 10.1016/j.eimc.2011.04.003.

13.- Wani R A, Malik A A, Chowdri N A, Wani K A, Naqash S H. Primary extrahepatic abdominal hydatidosis. Int J Surg. 2005; 3: 125-7. doi: 10.1016/j.ijsu.2005.06.004.

14.- Durgun V, Kapan S, Kapan M, Karabiçak I, Aydogan F, Goksoy E. Primary splenic hydatidosis. Dig Surg. 2003; 20: 38-41. doi: 10.1159/000068864.

15.- Eris C, Akbulut S, Yildiz M K, Abuoglu H, Odabasi M, Ozkan E, et al. Surgical approach to splenic hydatid cyst: single center experience. Int Surg. 2013; 98: 346-53. doi: 10.1007/s11605-012-1837-2.

16.- Ben Ameur H, Affes N, Abdelhedi C, Kchaou A, Boujelbene S, Beyrouti M I. Hydatid cyst of the spleen: Tunisian series of 21 cases. Indian J Surg. 2015; 77 (Suppl 2): 515-9. doi: 10.1007/ s11605-012-1837-2.

17.- Zhuoli Z, Yu Z, Liya X, Mingzhong L, Shengwei L. Case report: laparoscopic excision of a primary giant splenic hydatid cyst: literature review. Am J Trop Med Hyg. 2019;
101: 821-7. doi: 10.1007/s11605-012-1837-2.

18.- Manterola C, Otzen T. Checklist for reporting results using observational descriptive studies as research designs. The MInCir initiative. Int J Morphol. 2017; 35: 72-6. doi: 10.4067/S071795022017000100013.

19.- WHO Informal Working Group. International classification of ultrasound images in cystic echinococcosis for application in clinical and field epidemiological settings. Acta Trop. 2003; 85: 253-61. doi: 10.1016/s0001706x(02)00223-1.

20.- Clavien P A, Barkun J, de Oliveira M L, Vauthey J N, Dindo D, Schulick R D, et al. The Clavien-Dindo classification of surgical complications: five-year experience. Ann Surg 2009; 250: 187-96. doi: 10.1097/ SLA.0b013e3181b13ca2.

21.- Manterola C, Muñoz S, Fernández $\mathrm{O}$, Molina E, Barroso M. Laparotomía en J: una vía de acceso opcional para cirugía abdominal alta. Rev Chil Cir. 1999; 51: 275-82. http:// bases.bireme.br/cgi-bin/wxislind.exe/iah/ online/?IsisScript $=\mathrm{iah} / \mathrm{iah} . \mathrm{xis} \& \mathrm{src}=$ google $\&$ bas $\mathrm{e}=$ LILACS\&lang $=$ \&\&nextAction=lnk\&exprSea $\mathrm{rch}=245501 \&$ indexSearch $=$ ID

22.- Helsinki Statement. WMA Declaration of Helsinki - Ethical Principles for Medical Research Involving Human Subjects. 64 ${ }^{\text {th }}$ WMA General Assembly, Fortaleza, Brazil, October 2013. [Cited 22 Dec 2018]. Disponible en URL: https://www.wma.net/policies-post/ wma-declaration-of-helsinki-ethical-principlesfor-medical-research-involving-humansubjects.

23.- Ammann R W, Eckert J. Cestodes. Echinococcus. Gastroenterol Clin North Am 1996; 25: 655-89. doi: 10.1016/s08898553(05)70268-5.

24.- Franquet T, Montes M, Lecumberri F J, 
Esparza J, Bescos J M. Hydatid disease of the spleen: imaging findings in nine patients. Am J Roentgenol 1990; 154: 525-8. doi: 10.2214/ ajr.154.3.2106214.

25.- Bellakhdar A, Lamhamdi A, Touzani K, Khaiz D, Lakhloufi A, Bouzidi A, Diouri A. Hydatid cysts of the spleen. Apropos of 25 cases. J Chir. (Paris) 1986; 123: 326-9. PMID: 3745318.

26.- Fernández-Ruiz M, Guerra-Vales J M, EnguitaValls A B, Vila-Santos J, García-Borda F J, Morales-Gutiérrez C. Splenic hydatid cyst, a rare location of extrahepatic echinococcosis: Report of six cases. Eur J Intern Med. 2008; 19: e51-3. doi: 10.1016/j.ejim.2008.02.003.

27.- Nooghabi A J, Raoufian K, Motie M R. Concomitant splenic and hepatic hydatidosis: report of two cases and review of the literature. Acta Med Iran 2015; 53: 74-7. PMID: 25597610.

28.- Culafic D M, Kerkez M D, Mijac D D, Lekic N S, Rankovic V I, Lekic D D, et al. Spleen cystic echinococcosis: clinical manifestations and treatment. Scand J Gastroenterol. 2010; 45: 186-90. doi: 10.3109/00365520903428598.

29.- Safioleas M, Misiakos E, Manti C. Surgical treatment for splenic hydatidosis. World J Surg. 1997; 21: 374-7. doi: 10.1007/s002689900643.

30.- Meimarakis G, Grigolia G, Loehe F, Jauch K W, Schauer R J. Surgical management of splenic echinococcal disease. Eur J Med Res. 2009; 14: 165-70. doi: 10.1186/2047-783x-144-165.

31.- Ousadden A, Raiss M, Hrora A, AitLaalim S, Alaoui M, Sabbah F, et al. Kystes hydatiques de la rate: chirurgie radicale ou conservatrice? Pan Afr Med J. 2010; 5: 21. PMID: 21293748

32.- Tarcoveanu E, Bradea C, Vasilescu A, Georgescu S, Palaghia M, Crumpei F, et al. Splenic hydatid cyst: open or laparoscopic approach? J Surgery. 14: 39-44. Disponible en URL: https://www.hilarispublisher.com/ open-access/splenic-hydatid-cyst-open-orlaparoscopic-approach-10-1584-9341-14-1-6. pdf.

33.- Costi R, Castro Ruiz C, Zarzavadjian le Bian A, Scerrati D, Santi C, Violi V. Spleen hydatidosis treated by hemi-splenectomy: A low-morbidity, cost-effective management by a recently improved surgical technique. Int J Surg. 2015; 20: 41-5. doi: 10.1016/j.ijsu.2015.06.018.

34.- Vasilescu C, Tudor S, Popa M, Tiron A, Lupescu I. Robotic partial splenectomy for hydatid cyst of the spleen. Langenbecks Arch Surg. 2010; 395: 1169-74. doi: 10.1007/s00423010-0647-9.

35.- Esposito F, Noviello A, Moles N, Cantore N, Baiamonte M, Coppola Bottazzi E, et al. Partial splenectomy: a case series and systematic review of the literature. Ann Hepatobiliary Pancreat Surg. 2018; 22: 116-27. doi: 10.14701/ ahbps.2018.22.2.116

36.- Manciu S, Tudor S, Vasilescu C. Splenic cysts: A strong indication for a minimally invasive partial splenectomy. could the splenic hilar vasculature type hold a defining role? World J Surg. 2018; 41: 3543-50. doi: 10.1007/s11605012-1837-2.

37.- Ormeci N, Soykan I, Palabiyikoglu M, Idilman $\mathrm{R}$, Erdem H, Bektas A, et al. A new therapeutic approach for treatment of hydatid cysts of the spleen. Dig Dis Sci. 2002; 47: 2037-44. doi: 10.1023/a:1019672828967.

38.- Atmatzidis K, Papaziogas B, Mirelis C, Pavlidis T, Papaziogas T. Splenectomy versus spleen-preserving surgery for splenic echinococcosis. Dig Surg. 2003; 20: 527-31. doi: 10.1007/s11605-012-1837-2.

39.- Manterola C, Mansilla J A, Fonseca F, Preoperative albendazole and scolices viability in patients with hepatic echinococcosis. World J Surg. 2005; 29: 750-3. doi: 10.1007/s00268005-7691-6.

40.- Manterola C, Vial M, Losada H, Fonseca F, Bustos L, Muñoz S, Barroso M. Uncommon locations of abdominal hydatid disease. Trop Doct. 2003; 33: 179-80. doi: 10.1177/004947550303300324. 